\title{
Supply Chain Coordination with Market Scale Disruptions
}

\author{
Xiao-Bo Li \\ School of Statistics and Mathematics, Zhongnan University of Economics and Law, P.R. China, 430073 \\ Email: 276676455@qq.com
}

\begin{abstract}
This paper considers the scale of market demand and retailer's purchase costs disruptions in the disruption management of supply chain. When demand is a linear function of the price, the manufacturer's cost of production is a convex of retailers deviate at the same time, a disruption strategy is proposed to optimize the supply chain profit. When manufacture is the Stackelberg game leader, the coordination mechanism is proposed to coordinate the supply chain so as to realize the maximal profit of supply chain.
\end{abstract} scale

Key words: Supply chain coordination, Disruption, Market

\section{INTRODUCTION}

Enterprises are facing an increasingly severe pressure on the survival and development. Traditional management model has been unable to meet the ever-changing market demand and people have gradually come to realize supply chain management is an important means to enhance the competitiveness of enterprises, an effective management model to enhance the enterprise's adaptability to global competition. Being an important part of supply chain management, research of supply chain coordination has a great practical significance so as to improve supply chain performance.

Most of papers of disruption management of supply chain were based on the assumption that only a single factor was disrupted. But in practice, the unexpected event will inevitably lead to a number of factors disruptions in the supply chain system. This paper considers the scale of market demand and retailer's purchase costs disruptions in the disruption management of supply chain. When demand is a linear function of the price, the manufacturer's cost of production is a convex of retailers deviate at the same time, a disruption strategy is proposed to optimized the supply chain profit. When manufacture is the Stackelberg game leader, the coordination mechanism is proposed to coordinate the supply chain so as to realize the maximal profit of supply chain.

\section{II.SUPPLY CHAIN COORDINATION WITH MARKET SCALE}

After the manufacturer arranges the production plan, the emergency event can not only change the requirement of market but also change retailer's purchase costs. Suppose $a>0$ express market demand scale, $k>0$ expresses price sensitivity coefficient, and $c>0$ expresses retailer's purchase costs. Let $\Delta a$ be variable of the requirement, $\Delta c$ be variable of retailer's purchase costs, and $C_{r}$ be retailer's purchase assistant costs. Iff $a+\Delta a>0, c_{r}+\Delta c>0$, the assumption has the signification of fact.

Suppose $\bar{q}$ be the requirement of market after emergency event, $\bar{q}=a+\Delta a-k \bar{p}$. Let the difference between requirement $\bar{q}$ and the manufacturer's form production project $q^{*}$ be $\Delta q=\bar{q}-q^{*}$, when $\Delta q=\bar{q}-q^{*}<0$, production has some surplus, the surplus need to be sold in low price of secondary market; when $\Delta q=\bar{q}-q^{*}>0$, the more production need to be produced to satisfy with the new requirement. Certainly since new resource need to be used and manufacturer's production project has to be changed, the increase and surplus bring the incidental expenses.

After the emergency event, for the requirement $\bar{q}$, consider the profit function of supply chain on production disruption cost:

$$
\begin{aligned}
& \bar{f}^{s c}=\bar{q}\left(\frac{a+\Delta a-\bar{q}}{k}-c_{r}-\Delta c\right)-C(\bar{q})-\lambda_{1}\left(\bar{q}-q^{*}\right)^{+}-\lambda_{2}\left(q^{*}-\bar{q}\right)^{+} \text {(1) } \\
& \lambda_{1}, \lambda_{2}>0,(x)^{+}=\max (0, x), \lambda_{1} \text { expresses the }
\end{aligned}
$$

unit incidental cost of exceeding the form production; $\lambda_{2}$ expresses the unit incidental cost of reducing the form production. Since the surplus production should be sold in the lower price of marginal cost $C^{\prime}(\bar{q})$ in the secondary market, $0<\lambda_{2}<C^{\prime}(\bar{q})$

To account for the influence of market scale and retailer's purchase costs disruptions, we give theory 2.1:

Theory 2.1 In the instance of market scale and retailer's purchase costs disruptions of supply chain, suppose $\bar{q}^{*}$ expresses the optimal order quantity of the maximal profit function $\bar{f}^{s c} \quad$ of supply chain, (a) when
$\Delta a \geq k \Delta c, \bar{q}^{*} \geq \bar{q} ; \quad$ (b) when $\Delta a \leq k \Delta c, \bar{q}^{*} \leq \bar{q}$.
Prove (reduction to absurdity) : suppose when 
$\Delta a \geq k \Delta c, \bar{q}^{*}<\bar{q}, \bar{q}^{*}$ is the optimization of (1), in another words, $\bar{q}^{*}$ is the optimal solution of (2):

$$
\bar{f}_{0}^{s c}(\bar{q})=\bar{q}\left(\frac{a+\Delta a-\bar{q}}{k}-c_{r}-\Delta c\right)-C(\bar{q})-\lambda_{2}\left(q^{*}-\bar{q}\right)
$$

$$
\begin{aligned}
& \text { When } \begin{array}{c}
q^{*} \\
2 \bar{q}^{*}=a+\Delta a-k\left(c_{r}-\Delta c+C^{\prime}\left(\bar{q}^{*}\right)-\lambda_{2}\right)
\end{array}
\end{aligned}
$$$$
\bar{f}_{0}^{s c}(\bar{q})
$$$$
\text { acquires the maximal value. }
$$$$
\bar{q}^{*}-q^{*}=\frac{1}{2}\left(\Delta a-k \Delta c-k\left(C^{\prime}\left(\bar{q}^{*}\right)-C^{\prime}\left(q^{*}\right)\right)+k \lambda_{2}\right)
$$

$C(q)$ is the convex function, $C^{\prime \prime}(q)>0$, so $C^{\prime}(q)$ is incremental function. When $\bar{q}^{*}<q^{*}$, $C^{\prime}\left(\bar{q}^{*}\right)<C^{\prime}\left(q^{*}\right)$

Since

$\Delta a \geq k \Delta c,(k+\Delta k) \lambda_{2}>0$

$$
\bar{q}^{*}-q^{*}=\frac{1}{2}\left(\Delta a-k \Delta C-k\left(C^{\prime}\left(\bar{q}^{*}\right)-C^{\prime}\left(q^{*}\right)\right)+k \lambda_{2}\right) \geq 0
$$

So $\bar{q}^{*} \geq q^{*}$. This is contrary to the assumption, and the assumption is wrong.

Therefore when $\Delta a \geq k \Delta c, \bar{q}^{*} \geq \bar{q}$. In the same method, we can prove when $\Delta a \leq k \Delta c, \bar{q}^{*} \leq \bar{q}$.

Theory 2.1 demonstrates when market scale and retailer's purchase costs change in the same time, if the market demand increase and retailer's purchase costs decrease, $\Delta a \geq k \Delta c$, the manufacturer should increase production level to satisfy with market demand. So the manufacturer order more production to acquire the maximal profit; if the market demand decrease and retailer's purchase costs increase, $\Delta a \leq k \Delta c$, the manufacturer should decrease production level. When market scale and retailer's purchase costs increase or decrease in the same time, the market demand is not fix. The manufacturer shoud make production project and decide the optimal order quantity depending on theory 2.1 .

Concluding from theory 2.1, when $\Delta a \geq k \Delta c, \bar{q}^{*} \geq \bar{q}$ and market scale and retailer's purchase costs disrupt in the same time, the object function (1) can be predigested to:

$$
\bar{f}_{1}^{s c}(\bar{q})=\bar{q}\left(\frac{a+\Delta a-\bar{q}}{k}-c_{r}-\Delta c\right)-C(\bar{q})-\lambda_{1}\left(\bar{q}-q^{*}\right)
$$

We can prove easily $\bar{f}_{1}^{s c}(\bar{q})$ is concave function about $\bar{q}$. So $\bar{f}_{1}^{\text {sc }}(\bar{q})$ need to have optimal solutions. Not considering the condition $\bar{q}^{*} \geq \bar{q}$, the optimal solution is:

$$
2 \bar{q}^{*}=a+\Delta a-k\left(c_{r}+\Delta C+C^{\prime}\left(\bar{q}_{1}\right)+\lambda_{1}\right)
$$

(2-7) subtracts (2-3):

$$
2\left(\bar{q}-q^{*}\right)+k\left(C^{\prime}\left(\bar{q}_{1}\right)-C^{\prime}\left(q^{*}\right)\right)=\Delta a-k \Delta C-k \lambda_{1}
$$

$C(q)$ is the convex function, $C^{\prime \prime}(q)>0$, so $C^{\prime}(q)$ is incremental function, and

when

$$
\begin{aligned}
& \bar{q}_{1} \geq q^{*}, 2\left(\bar{q}-q^{*}\right)+k\left(C^{\prime}\left(\bar{q}_{1}\right)-C^{\prime}\left(q^{*}\right)\right) \geq 0 . \\
& \bar{q}_{1} \leq q^{*}, 2\left(\bar{q}-q^{*}\right)+k\left(C^{\prime}\left(\bar{q}_{1}\right)-C^{\prime}\left(q^{*}\right)\right) \leq 0 .
\end{aligned}
$$

\section{OPTIMIZE THE SUPPLY CHAIN PROFIT DISRUPTION STRATEGY}

The case 1: $\quad \Delta a-k \Delta c>k \lambda_{1}$

In the situation, $\bar{q}_{1}$ satisfies with the condition $\bar{q}^{*} \geq q^{*} \quad$ so $\bar{q}_{1}$ is the optimal solution

$\bar{q}_{1}^{*}=\bar{q}_{1}=\frac{a+\Delta a-k\left(c_{r}+\Delta C+C^{\prime}\left(\bar{q}_{1}^{*}\right)+\lambda_{1}\right)}{2}$

The corresponding optimal retail price is: $\bar{p}_{1}^{*}=\frac{a+\Delta a-\bar{q}_{1}^{*}}{k}$

chain is:

(10), and the maximal profit of supply

$$
\begin{gathered}
\bar{f}_{1}^{s c^{*}}=\left(\bar{p}_{1}^{*}-c_{r}-\Delta c\right) \bar{q}_{1}^{*}-\lambda_{1}\left(\bar{q}_{1}-q^{*}\right) \\
=\bar{f}^{s^{*}}+\left(\frac{\Delta a-k \Delta c}{k}\right) q^{*}-\left(C\left(\bar{q}_{1}^{*}\right)-C\left(q^{*}\right)\right)+\frac{\left(\Delta a-k \Delta c-k\left(\lambda_{1}-C\left(q^{*}\right)\right)\right)^{2}-\left(k C\left(\bar{q}^{*}\right)\right)^{2}}{4 k}
\end{gathered}
$$

Concluding from (9) to (11), when $\Delta a-k \Delta c>k \lambda_{1}$, the optimal order quantity, optimal retail price and the maximal profit of supply chain are affected by market scale and retailer's purchase costs.

The case 2: $\Delta a-k \Delta c \leq k \lambda_{2}$

Similar with above analysis, the optimal order quantity of the two situations: 
$q^{*}=\left\{\begin{array}{cl}\bar{q}_{3}^{*}=q^{*}, & k \lambda_{2} \leq \Delta a-k \Delta c \leq 0 \\ \bar{q}_{4}^{*}=\frac{a+\Delta a-k\left(c_{r}+\Delta c+C^{\prime}\left(\bar{q}_{4}\right)-\lambda_{2}\right)}{2}, & \Delta a-k \Delta c \leq k \lambda_{2}\end{array}\right.$

The corresponding optimal retail price is:

$\bar{p}^{*}=\left\{\begin{array}{cl}\bar{p}_{3}{ }^{*}=p^{*}+\frac{\Delta a}{k}, & k \lambda_{2} \leq \Delta a-k \Delta c \leq 0 \\ \bar{p}_{4}{ }^{*}=\frac{a+\Delta a+k\left(c_{r}+\Delta c+C^{\prime}\left(\bar{q}_{4}\right)-\lambda_{2}\right)}{2 k}, & \Delta a-k \Delta c \leq k \lambda_{2}\end{array}\right.$

The maximal profit of supply chain in the two situation:

$$
\begin{gathered}
\bar{f}_{3}^{s c^{*}}=f^{s c^{*}}+\frac{\Delta a-k \Delta c}{k} q^{*} \\
\bar{f}_{4}^{s c^{*}}=\left(\bar{p}_{4}^{*}-c_{r}-\Delta c\right) \bar{q}_{4}^{*}-\lambda_{1}\left(\bar{q}_{4}-q^{*}\right) \\
=\bar{f}^{c^{\prime}}+\left(\frac{\Delta a-k \Delta c}{k}\right) q^{*}-\left(c\left(\bar{q}_{4}^{*}\right)-c\left(q^{*}\right)\right)+\frac{\left(\Delta a-k \Delta c-k\left(\lambda_{2}-c\left(q^{*}\right)\right)\right)^{2}-\left(k c\left(\bar{q}_{4}^{*}\right)\right)^{2}}{4 k}
\end{gathered}
$$

\section{CONCLUSION}

In the situation of market scale and retailer's purchase costs disruptions at the same time, when the transformation of $|\Delta a-k \Delta C|$ is small (satisfying with $k \lambda_{2} \leq \Delta a-k \Delta c \leq 0$ ), maintain the production project before, and recuperate demand disruption cost according to adjust retail price. In the circumstance, retail price is only affected by market scale which is increasing with the development of market scale. When the transformation of $|\Delta a-k \Delta c|$ is large, the production project need to be adjusted. For retailer price, in spite of magnitude of the transformation about $|\Delta a-k \Delta C|$, as long as market demand changes, retail price need to be adjusted: if market demand scale develops, the retail price increases; if market demand scale shrinks , the retail price decreases.

\section{REFERENCES}

[1] Yu H,Chen J, Yu G. How to coordinate supply chain under Disruption. System Engineering Theory and Practice, 7(2005), p9-16.

[2] Tomlin B. On the value of mitigation and contingency strategies for managing supply chain disruption risks. Management Science,5 (2006), p639-657.

[3] C. Schneeweiss, K. Zimmer, M. Zimmermann. The design of contracts to coordinate operational interdependencies within the supply chain[J]. International Journal of Production Economics, 1(2004), p43-59. 